\title{
How well can one resolve the state space of a chaotic map?
}

\author{
Domenico Lippolis and Predrag Cvitanović \\ Center for Nonlinear Science, School of Physics, \\ Georgia Institute of Technology, Atlanta, GA 30332-0430
}

(Dated: May 23, 2022)

\begin{abstract}
All physical systems are affected by some noise that limits the resolution that can be attained in partitioning their state space. For chaotic, locally hyperbolic flows, this resolution depends on the interplay of the local stretching/contraction and the smearing due to noise. We propose to determine the 'finest attainable' partition for a given hyperbolic dynamical system and a given weak additive white noise, by computing the local eigenfunctions of the adjoint Fokker-Planck operator along each periodic point, and using overlaps of their widths as the criterion for an optimal partition. The Fokker-Planck evolution is then represented by a finite transition graph, whose spectral determinant yields time averages of dynamical observables. Numerical tests of such 'optimal partition' of a onedimensional repeller support our hypothesis.
\end{abstract}

PACS numbers: $05.45 .-\mathrm{a}, 45.10 . \mathrm{db}, 45.50 . \mathrm{pk}, 47.11 .4 \mathrm{j}$

The effect of noise on the behavior of a nonlinear dynamical system is a fundamental problem in many areas of science $[1,2,3]$, and the interplay of noise and chaotic dynamics is of particular current interest $[4,5,6]$.

The purpose of this letter is two-fold. First, and conceptually the most important, we point out an effect of noise that has not been addressed in literature: weak noise limits the attainable resolution of the state space ('phase space') of a chaotic system. We formulate the 'optimal partition' hypothesis whose implementation requires only integration of a small set of solutions of the deterministic equations of motion. Second, more technical point; we show that the optimal partition hypothesis replaces the Fokker-Planck PDEs by finite, low-dimensional Fokker-Planck matrices, whose eigenvalues give good estimates of long-time observables (escape rates, Lyapunov exponents, etc.).

A chaotic trajectory explores a strange attractor, and evaluation of long-time averages requires effective partitioning of the state space into smaller regions. The set of unstable periodic orbits forms a 'skeleton' that can be used to partition the state space into such smaller regions, each region a neighborhood of a periodic point $[7,8]$ (i.e., a point on a periodic orbit). The number of periodic orbits grows exponentially with period length, yielding finer and finer partitions, with the neighborhood of each periodic orbit shrinking exponentially.

As there is an infinity of periodic orbits, with each neighborhood shrinking asymptotically to a point, a deterministic chaotic system can - in principle - be resolved arbitrarily finely. However, any physical system suffers background noise, any numerical prediction suffers computational roundoff noise, and any set of equations models nature up to a given accuracy, since degrees of freedom are always neglected. If the noise is weak, the short-time dynamics is not altered significantly: short periodic orbits of the deterministic flow still partition coarsely the state space. Intuitively, the noise smears out the neigh- borhood of a periodic point, whose size is now determined by the interplay between the diffusive spreading parameterized $[9,10]$ by the diffusion constant $D$, and its exponentially shrinking deterministic neighborhood. As the periods of periodic orbits increase, the diffusion always wins, and successive refinements of a deterministic partition of the state space stop at the finest attainable partition, beyond which the diffusive smearing exceeds the size of any deterministic subpartition. The smearing width differs from trajectory to trajectory, so there is no one single time beyond which noise takes over; rather, as we shall show here, the optimal partition has to be computed for a given dynamical system and given noise. This effort brings a handsome practical reward: as the optimal partition is finite, the Fokker-Planck operator can be represented by a finite matrix.

While the general idea is intuitive, nonlinear dynamics interacts with noise in a nonlinear way, and methods for implementing the optimal partition for a given noise still need to be developed. In this letter we propose a new approach to this partitioning. We compute the width of the leading eigenfunction of the linearized adjoint FokkerPlanck operator on each periodic point. The optimal partition is then obtained by tracking the diffusive widths of unstable periodic orbits until they start to overlap. We describe here the approach as applied to $1 d$ expanding maps; higher-dimensional hyperbolic maps and flows require a separate treatment for contracting directions, a topic for a future publication [11].

As the simplest application of the method, consider the orbit $\left\{\ldots, x_{-1}, x_{0}, x_{1}, x_{2}, \ldots\right\}$ of a $1 d \operatorname{map} x_{n+1}=f\left(x_{n}\right)$, and the associated discrete Langevin equation [2]

$$
x_{n+1}=f\left(x_{n}\right)+\xi_{n},
$$

where the $\xi_{n}$ are independent Gaussian random variables of mean 0 and variance $2 D$ (the method can be applied to continuous time flows as well, but a $1 d$ map suffices to illustrate the optimal partition algorithm). The cor- 
responding Fokker-Planck operator [3],

$$
\mathcal{L} \circ \rho_{n}(y)=\int \frac{d x}{\sqrt{4 \pi D}} e^{-\frac{(y-f(x))^{2}}{4 D}} \rho_{n}(x)
$$

carries the density of Langevin trajectories $\rho_{n}(x)$ forward in time to $\rho_{n+1}=\mathcal{L} \circ \rho_{n}$. Since a density concentrated at point $x_{n}$ is carried into a density concentrated at $x_{n+1}$, we introduce local coordinate systems $z_{a}$ centered on the orbit points $x_{a}$, together with a notation for the map (1), its derivative, and, by the chain rule, the derivative of the $k$ th iterate $f^{k}$ evaluated at the point $x_{a}$,

$$
\begin{aligned}
x & =x_{a}+z_{a}, \quad f_{a}\left(z_{a}\right)=f\left(x_{a}+z_{a}\right) \\
f_{a}^{\prime} & =f^{\prime}\left(x_{a}\right), \quad f_{a}^{k \prime}=f_{a+k-1}^{\prime} \cdots f_{a+1}^{\prime} f_{a}^{\prime}, \quad k \geq 2 .
\end{aligned}
$$

Here $a$ is the label of point $x_{a}$, and the label $a+1$ is a shorthand for the next point $b$ on the orbit of $x_{a}, x_{b}=$ $x_{a+1}=f\left(x_{a}\right)$. For example, a period-3 periodic point might have label $a=001$, and by $x_{010}=f\left(x_{001}\right)$ the next point label is $b=010$.

If the noise is weak, we can approximate (to leading order in $D)$ the Fokker-Planck operator, $\mathcal{L}_{a} \circ \rho_{n}\left(x_{a+1}+\right.$ $\left.z_{a+1}\right)=\int d z_{a} \mathcal{L}_{a}\left(z_{a+1}, z_{a}\right) \rho_{n}\left(x_{a}+z_{a}\right)$, by linearization centered on $x_{a}$, the ath point along the orbit,

$$
\mathcal{L}_{a}\left(z_{a+1}, z_{a}\right)=(4 \pi D)^{-1 / 2} e^{-\frac{\left(z_{a+1}-f_{a}^{\prime} z_{a}\right)^{2}}{4 D}} .
$$

$\mathcal{L}_{a}$ maps a Gaussian density $\rho_{n}\left(x_{a}+z_{a}\right)=$ $c_{a} \exp \left\{-z_{a}^{2} / 2 \sigma_{a}^{2}\right\}$, of variance $\sigma_{a}^{2}$, into a Gaussian density $\rho_{n+1}(x)$ of variance $\sigma_{a+1}^{2}=\left(f_{a}^{\prime} \sigma_{a}\right)^{2}+2 D$. This variance is an interplay of the Brownian noise contribution $2 D$ and the nonlinear contracting/amplifying contribution $\left(\sigma f^{\prime}\right)^{2}$. The diffusive dynamics of a nonlinear system are thus fundamentally different from Brownian motion, as the map induces a history dependent effective noise.

In order to determine the smallest noise-resolvable state space partition along the trajectory of $x_{a}$, we need to determine the effect of noise on the points preceding $x_{a}$. This is achieved by the adjoint Fokker-Planck operator

$$
\mathcal{L}^{\dagger} \circ \tilde{\rho}_{n}(x)=\int \frac{d y}{\sqrt{4 \pi D}} e^{-\frac{(y-f(x))^{2}}{4 D}} \tilde{\rho}_{n}(y),
$$

which relates a density $\tilde{\rho}_{n}$ concentrated around $x_{a}$ to $\tilde{\rho}_{n-1}=\mathcal{L}^{\dagger} \circ \tilde{\rho}_{n}$, a density concentrated around the previous point $x_{a-1}$, the variance transforming as $\left(f_{a-1}^{\prime} \sigma_{a-1}\right)^{2}=\sigma_{a}^{2}+2 D$. For an unstable (expanding) map, these variances shrink. After $n$ steps the variance is given by

$$
\left(f_{a-n}^{n^{\prime}} \sigma_{a-n}\right)^{2}=\sigma_{a}^{2}+2 D\left(1+\left(f_{a-1}^{\prime}\right)^{2}+\cdots+\left(f_{a-n+1}^{n-1^{\prime}}\right)^{2}\right) .
$$

From the dynamical point of view, a good state space partition encodes the recurrent dynamics; here we shall seek a partition in terms of neighborhoods of periodic points $[8,12]$ of short periods. For the linearized $\mathcal{L}_{a}^{\dagger}$

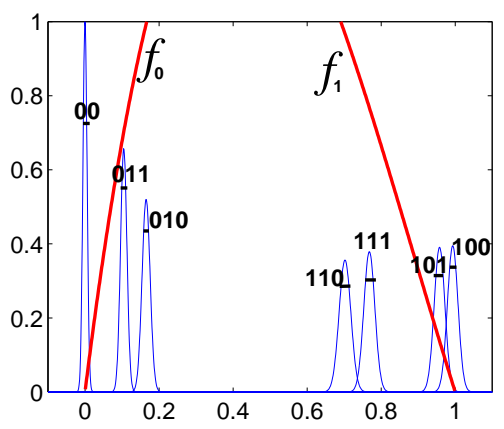

FIG. 1: $f_{0}, f_{1}$ : branches of the deterministic map (9) for $\Lambda_{0}=8$ and $b=0.6$. The local eigenfunctions $\tilde{\rho}_{a, 0}$ with variances given by (8) provide a state space partitioning by neighborhoods of periodic points of period 3. These are computed for noise variance ( $D=$ diffusion constant) $2 D=0.002$. The neighborhoods $\mathcal{M}_{000}$ and $\mathcal{M}_{001}$ already overlap, so $\mathcal{M}_{00}$ cannot be resolved further. For periodic points of period 4, only $\mathcal{M}_{011}$ can be resolved further, into $\mathcal{M}_{0110}$ and $\mathcal{M}_{0111}$.

acting on a fixed point $x_{a}=f\left(x_{a}\right)$, the $n \rightarrow \infty$ sum (6) converges to a Gaussian of variance

$$
\sigma_{a}^{2}=2 D /\left(\Lambda_{a}^{2}-1\right),
$$

where $\Lambda_{a}=f_{a}^{\prime}$, and for a periodic point $x_{a} \in p$ to a Gaussian of variance

$$
\sigma_{a}^{2}=\frac{2 D}{1-\Lambda_{p}^{-2}}\left(\frac{1}{\left(f_{a}^{\prime}\right)^{2}}+\cdots+\frac{1}{\Lambda_{p}^{2}}\right)
$$

where $\Lambda_{p}=f_{a}^{n_{p \prime}}$ is the Floquet multiplier (eigenvalue of the Jacobian linearized flow) of an unstable $\left(\left|\Lambda_{p}\right|>1\right)$ periodic orbit $p$ of period $n_{p}$. This is the key formula; note that its evaluation requires no Fokker-Planck formalism, it depends only on the deterministic orbit and its linear stability.

We can now state the main result of this letter, 'the best possible of all partitions' hypothesis, as an algorithm: assign to each periodic point $x_{a}$ a neighborhood of finite width $\left[x_{a}-\sigma_{a}, x_{a}+\sigma_{a}\right]$. Consider periodic orbits of increasing period $n_{p}$, and stop the process of refining the state space partition as soon as the adjacent neighborhoods overlap.

As a concrete application to the Langevin map (1) consider map [12]

$$
f(x)=\Lambda_{0} x(1-x)(1-b x)
$$

plotted in figure 1 ; this figure also shows the local eigenfunctions $\tilde{\rho}_{a, 0}$ with variances given by (8). Each Gaussian is labeled by the $\left\{f_{0}, f_{1}\right\}$ branches visitation sequence of the corresponding deterministic periodic point (a symbolic dynamics, however, is not a prerequisite for implementing the method). We find that in this case the state space (the unit interval) can be resolved into 7 neighborhoods

$$
\left\{\mathcal{M}_{00}, \mathcal{M}_{011}, \mathcal{M}_{010}, \mathcal{M}_{110}, \mathcal{M}_{111}, \mathcal{M}_{101}, \mathcal{M}_{100}\right\} .
$$




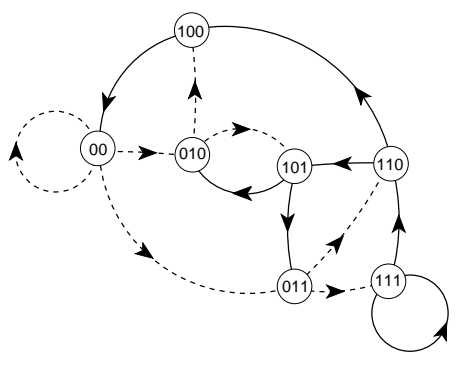

FIG. 2: Transition graph (graph whose links correspond to the nonzero elements of a transition matrix $T_{b a}$ ) describes which regions $b$ can be reached from the region $a$ in one time step. The 7 nodes correspond to the 7 regions of the optimal partition (10). Dotted links correspond to symbol 0, and the full ones to 1 , indicating that the next region is reached by the $f_{0}$, respectively $f_{1}$ branch of the map plotted in figure 1 .

Evolution in time maps the optimal partition interval $\mathcal{M}_{011} \rightarrow\left\{\mathcal{M}_{110}, \mathcal{M}_{111}\right\}, \mathcal{M}_{00} \rightarrow\left\{\mathcal{M}_{00}, \mathcal{M}_{011}, \mathcal{M}_{010}\right\}$, etc., as compactly summarized by the transition graph of figure 2 .

Next we show that the optimal partition enables us to replace Fokker-Planck PDEs by finite-dimensional matrices. The variance (8) is stationary under the action of $\mathcal{L}_{a}^{\dagger n_{p}}$, and the corresponding Gaussian is thus an eigenfunction. Indeed, for the linearized flow the entire eigenspectrum is available analytically, and will be a key ingredient in what follows. For a periodic point $x_{a} \in p$, the $n_{p}$ th iterate $\mathcal{L}_{a}^{n_{p}}$ of the linearization (4) is the discrete time version of the Ornstein-Uhlenbeck process [13], with left $\tilde{\rho}_{0}, \tilde{\rho}_{1}, \cdots$, respectively right $\rho_{0}, \rho_{1}, \cdots$ mutually orthogonal eigenfunctions [3] given by

$$
\begin{aligned}
\tilde{\rho}_{a, k}(z) & =\frac{\beta^{k+1}}{\sqrt{\pi} 2^{k} k !} H_{k}(\beta z) e^{-(\beta z)^{2}} \\
\rho_{a, k}(z) & =\frac{1}{\beta^{k}} H_{k}(\beta z),
\end{aligned}
$$

where $H_{k}(x)$ is the $k$ th Hermite polynomial, $1 / \beta=$ $\sqrt{2} \sigma_{a}$, and the $k$ th eigenvalue is $1 /|\Lambda| \Lambda^{k}$.

Partition (10) being the finest possible partition, the Fokker-Planck operator now acts as $[7 \times 7]$ matrix with non-zero $a \rightarrow b$ entries expanded in the Hermite basis,

$$
\begin{aligned}
{\left[\mathbf{L}_{b a}\right]_{k j}=} & \left\langle\tilde{\rho}_{b, k}|\mathcal{L}| \rho_{a, j}\right\rangle \\
= & \int \frac{d z_{b} d z_{a} \beta}{2^{j+1} j ! \pi \sqrt{D}} e^{-\left(\beta z_{b}\right)^{2}-\frac{\left(z_{b}-f_{a}\left(z_{a}\right)\right)^{2}}{4 D}} \\
& \quad \times H_{k}\left(\beta z_{b}\right) H_{j}\left(\beta z_{a}\right),
\end{aligned}
$$

where $1 / \beta=\sqrt{2} \sigma_{a}$, and $z_{a}$ is the deviation from the periodic point $x_{a}$. It is the number of resolved periodic points that determines the dimensionality of the FokkerPlanck matrix.

Periodic orbit theory $[12,14]$ expresses the long-time dynamical averages, such as Lyapunov exponents, escape rates, and correlations, in terms of the leading eigenvalues of the Fokker-Planck operator $\mathcal{L}$. In our 'optimal

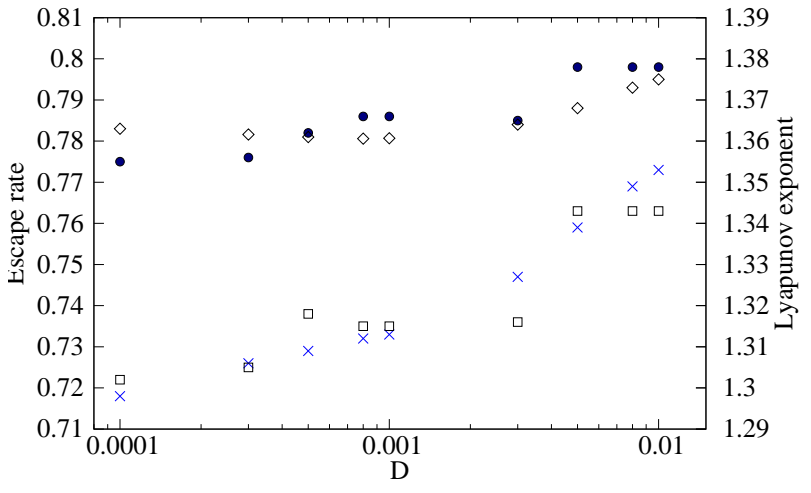

FIG. 3: (left scale) the escape rate of the repeller (9) vs. the noise strength $D$, calculated using $(\square)$ the 'optimal partition', and $(\times)$ a uniform discretization (16) in $N=128$ intervals; (right scale) the Lyapunov exponent of the same repeller vs. $D$, estimated using $(\bullet)$ the 'optimal partition', and $(\diamond)$ the average (17).

partition' approach, $\mathcal{L}$ is approximated by the finitedimensional matrix $\mathbf{L}$, and its eigenvalues are determined from the zeros of $\operatorname{det}(1-z \mathbf{L})$, expanded as a polynomial in $z$, with coefficients given by traces of powers of $\mathbf{L}$. As the trace of the $n$th iterate of the Fokker-Planck operator $\mathcal{L}^{n}$ is concentrated on periodic points $f^{n}\left(x_{a}\right)=x_{a}$, we evaluate the contribution of periodic orbit $p$ to $\operatorname{tr} \mathbf{L}^{n_{p}}$ by centering $\mathbf{L}$ on the periodic orbit,

$$
t_{p}=\operatorname{tr}_{p} \mathcal{L}^{n_{p}}=\operatorname{tr} \mathbf{L}_{a d} \cdots \mathbf{L}_{c b} \mathbf{L}_{b a},
$$

where $x_{a}, x_{b}, \cdots x_{d} \in p$ are successive periodic points. To leading order in the noise variance $2 D, t_{p}$ takes the deterministic value $t_{p}=1 /\left|\Lambda_{p}-1\right|$.

We illustrate the method by calculating the escape rate $\gamma=-\ln z_{0}$, where $z_{0}^{-1}$ is the leading eigenvalue of Fokker-Planck operator $\mathcal{L}$, for the repeller plotted in figure 1 . The spectral determinant can be read off the transition graph of figure 2 ,

$$
\begin{aligned}
& \operatorname{det}(1-z \mathbf{L})=1-\left(t_{0}+t_{1}\right) z-\left(t_{01}-t_{0} t_{1}\right) z^{2} \\
& \quad-\left(t_{001}+t_{011}-t_{01} t_{0}-t_{01} t_{1}\right) z^{3}-\cdots \\
& \quad-\left(t_{0010111}+t_{0011101}-\cdots+t_{001} t_{011} t_{1}\right) z^{7}
\end{aligned}
$$

The polynomial coefficients are given by products of nonintersecting loops of the transition graph [12], with the escape rate given by the leading root $z_{0}^{-1}$ of the polynomial. Twelve periodic orbits $\overline{0}, \overline{1}, \overline{01}, \overline{001}, \overline{011}, \overline{0011}, \overline{0111}$, $\overline{00111}, \overline{001101}, \overline{001011}, \overline{0010111}, \overline{0011101}$ up to period 7 (out of the 41 contributing to the noiseless, deterministic cycle expansion up to cycle period 7) suffice to fully determine the spectral determinant of the Fokker-Planck operator. The 'optimal partition' estimate of the Lyapunov exponent is given [12] by $\lambda=\langle\ln \Lambda\rangle /\langle n\rangle$, where the cycle expansion average of an observable $A$

$$
\begin{gathered}
\langle A\rangle=A_{0} t_{0}+A_{1} t_{1}+\left(A_{01} t_{01}-\left(A_{0}+A_{1}\right) t_{0} t_{1}\right)+ \\
\left(A_{001} t_{001}-\left(A_{01}+A_{0}\right) t_{01} t_{0}\right)+\cdots
\end{gathered}
$$


is the finite sum over cycles contributing to (14), and $\ln \Lambda_{p}=\sum \ln \left|f^{\prime}\left(x_{a}\right)\right|$ sum over the points of cycle $p$ is the cycle Lyapunov exponent.

Since our 'optimal partition' algorithm is based on a sharp overlap criterion, small changes in noise strength $D$ can lead to transition graphs of different topologies. We assess the accuracy of our finite Fokker-Planck matrix approximations by discretizing the Fokker-Planck operator $\mathcal{L}$ with a piecewise-constant approximation on a uniform mesh on the unit interval [15],

$$
[\mathcal{L}]_{i j}=\frac{1}{\sqrt{4 \pi D}} \int_{\mathcal{M}_{i}} \frac{d x}{\left|\mathcal{M}_{i}\right|} \int_{f^{-1}\left(\mathcal{M}_{j}\right)} d y e^{-\frac{1}{4 D}(y-f(x))^{2}}
$$

where $\mathcal{M}_{i}$ is the $i$ th interval in equipartition of the unit interval into $N$ pieces. Empirically, $N=128$ intervals suffice to compute the leading eigenvalue of the discretized $[128 \times 128]$ matrix $[\mathcal{L}]_{i j}$ to four significant digits. The Lyapunov exponent is evaluated as the average

$$
\lambda=\int d x e^{\gamma} \rho(x) \ln \left|f^{\prime}(x)\right|
$$

where $\rho(x)$ is the leading eigenfunction of (16), $\gamma$ is the escape rate, and $e^{\gamma} \rho$ is the normalized repeller measure, $\int d x e^{\gamma} \rho(x)=1$. The numerical results are summarized in figure 3, with the estimates of the 'optimal partition' method within $1 \%$ of those given by the uniform discretization of Fokker-Planck.

In summary, we have presented a new method for partitioning the state space of a chaotic repeller in the presence of noise. The key idea is that the width of the linearized adjoint Fokker-Planck operator $\mathcal{L}_{a}^{\dagger}$ eigenfunction computed on a periodic point $x_{a}$ provides the scale beyond which no further local refinement of state space is possible. This computation enables us to systematically determine the optimal partition, of the finest state space resolution attainable for a given chaotic dynamical system and a given noise. Once the optimal partition is determined, we use the associated transition graph to describe the stochastic dynamics by a finite dimensional Fokker-Planck matrix. While an expansion of the FokkerPlanck operator about periodic points was already introduced in ref. [16], the novel aspect of this work is its representation in terms of the eigenfunctions of the linearized Fokker-Planck operator (4), ie. the Hermite basis $[10,11]$.

We test our optimal partition hypothesis by applying it to evaluation of the escape rate and the Lyapunov expo- nent of a $1 d$ repeller in presence of additive noise. Numerical tests indicate that, the 'optimal partition' method can be as accurate as a 128-interval discretization of the Fokker-Planck operator.

The success of the optimal partition hypothesis in a 1-dimensional setting is encouraging. However, higherdimensional hyperbolic maps and flows, for which an effective optimal partition algorithm would be very useful, present new challenges due to the subtle interactions between expanding, marginal and contracting directions. The nonlinear diffusive effects (weak stochastic corrections [16]) need to be accounted for as well. These issues will be addressed in a future publication [11].

We are indebted to C.P. Dettmann, W.H. Mather, A. Grigo and G. Vattay for many stimulating discussions, and S.A. Solla for a critical reading of the manuscript. P.C. thanks Glen P. Robinson and NSF grant DMS0807574 for partial support.

[1] N. G. van Kampen, Stochastic Processes in Physics and Chemistry (North-Holland, Amsterdam, 1992).

[2] A. Lasota and M. MacKey, Chaos, Fractals, and Noise; Stochastic Aspects of Dynamics (Springer-Verlag, Berlin, 1994).

[3] H. Risken, The Fokker-Planck Equation (SpringerVerlag, 1996).

[4] P. Gaspard, J. Stat. Phys. 106, 57 (2002).

[5] H. C. Fogedby, Phys. Rev. Lett. 94, 195702 (2005).

[6] H. C. Fogedby, Phys. Rev. E 73, 031104 (2006).

[7] D. Ruelle, Statistical Mechanics, Thermodynamic Formalism (Addison-Wesley, Reading, MA, 1978).

[8] P. Cvitanović, Phys. Rev. Lett. 61, 2729 (1988).

[9] H. Dekker and N. V. Kampen, Physics Lett. 73A, 374 (1979).

[10] P. Gaspard, G. Nicolis, A. Provata, and S. Tasaki, Phys. Rev. E 51, 74 (1995).

[11] D. Lippolis and P. Cvitanović, in preparation, 2009.

[12] P. Cvitanović, R. Artuso, R. Mainieri, G. Tanner, and G. Vattay, Chaos: Classical and Quantum (Niels Bohr Institute, Copenhagen, 2009), ChaosBook.org.

[13] G. E. Uhlenbeck and L. S. Ornstein, Phys. Rev. 36, 823 (1930).

[14] P. Gaspard, Chaos, Scattering and Statistical Mechanics (Cambridge Univ. Press, Cambridge, 1997).

[15] S. M. Ulam, A Collection of Mathematical Problems (Interscience Publishers, New York, 1960).

[16] P. Cvitanović, N. Søndergaard, G. Palla, G. Vattay, and C. Dettmann, Phys. Rev. E 60, 3936 (1999). 